\title{
System Capability Estimation for Various Information Operations Used
}

\author{
Alexander Geyda ${ }^{1, *}$ \\ ${ }^{1}$ St. Petersburg Federal Research Center of the Russian Academy of Sciences
}

\begin{abstract}
The article considers system capability (or - system potential) estimation regarding different kinds of information operations use. Information operations classification suggested. System capability is introduced in the article as property to research teleological (goal- and effects-related) aspects of information technology. Such aspects are studied analytically, with predictive and prescriptive mathematical models and methods. Researchers can apply system capability to dynamic capability, organizational capability investigation regarding information technology, learning technology, and innovation technologies use. Example of system capability estimation considered.
\end{abstract}

\section{Introduction}

There is a gap between the need to decide a variety of problems, such as dynamic capability $[1,2]$, adaptability [3], resilience [4], IT-enabled capabilities [5-7], information technology (IT) value investigation problems [8-10], infonomics [11], IT governance problems [12], related to the evaluating the quality of system functioning in turbulent environments [13] and to the measurement of IT use, on the one hand, and theoretical models and methods available for such problems decision as related mathematical problems - on the other hand $[14,15]$. We suggest system capability analytical research methods and models to fill this gap. System capability is a system's ability to achieve changing goals as a reaction to turbulent environments [16]. We consider complex technical, technological, socio-technical systems (CTS), which are such systems that include interrelated elements of a different nature, i.e., mechanical, organizational, human, and technological components [17]. For such CTS, the capability is required: to react to CTS environment changes and impacts, to respond to environmental changes, attacks, to respond on goals changes [18]. So, system capability needed to interact appropriately with the environment and parts of the system under environmental impacts. Information operations are required to create such system capability and to provide interaction under changed conditions. The system capability is used to estimate dynamic capabilities indicators, organizational capabilities indicators, system dependability indicators, and IT-enabled capabilities indicators.

Further, indicators of this property are used to solve various practical problems as appropriate mathematical problems of indicators estimation and the CTS elements, capabilities, information operations synthesis based on indicators, estimated as a function of

* Corresponding author geida@iias.spb.su 
possible CTS characteristics. For assessment of such property complex of models is necessary. It shall reflect the interacting system, its environments of a different kind, and information operations. Information operations are required to check the system and its environment functioning states to measure their correspondence.

Then information operations are used to alternate the CTS functioning to achieve a possibly changed goal set by changing environment and with changing impact on the CTS. CTS's research in varying conditions and IT use to react to changes and provide quality interaction with the environment are traditionally implemented based on the pragmatic properties of such use in the functional domain $[19,20]$. The objects' pragmatic properties under study are properties of various objects, such that these properties characterize the results of the activity with objects. Pragmatic properties are measured by the effects (main results) of actions at the boundary and then by these effects' compliance with the environment's requirements. Activity is always implemented using certain information operations (at least using human senses and brain) to check state, to describe future actions, check the results, and change the action. Unfortunately, such activities are not yet studied in sufficient detail.

\section{Information Operations Classification and Quality Estimation}

Classification of information technology actions (operations) illustrated at figure 1. First level classification shown.

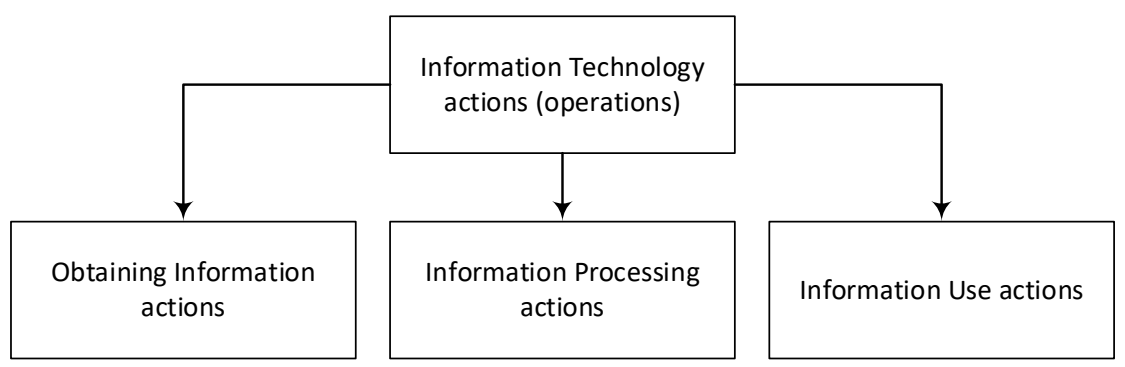

Fig.1. Information Technology actions classification. First-level classification.

The goal of the first two kinds of IT operations is information itself. Under information Im here, we mean the form of the existence of ideas or thoughts in the material world (that is, outside of mind). Such form, for example, is a record on some «information carrier.» Difference of actions to obtain information $a^{o}: M, m \rightarrow I m$ is their input $M m$ can be material world or mind, but information processing actions $a^{I p}: I m_{1} \rightarrow I m_{2}$ take information $I m_{1}$ as input and produce information as $I m_{2}$ as output. This information can be both prescriptive $\operatorname{Imp}$ or descriptive Imd or their system. Finally, information use actions $a^{I u}: I m \rightarrow M m$ take information $I m$ as input and produce changes outside $(M)$ or inside $(m)$ mind. Such changes are made by humans or devices (actuators, robots) using information $\operatorname{Imp}$ in case of information used outside mind and Imd or Imp if inside. Changes happen because information $I m$ may alter the way humans or devices interact with the material world $(M)$ or mind $(m)$. Thus, changes in $I m$ may cause changes in $M m$. This change is the effect of $I m$ use and the effect of IT use. IT describes operations $a^{j}$ of different kinds $j$ and their relations with other operations, with minds and material (outside minds) world. Thus, information technology describes possible IT operations and chains of information operations. First level classification provides a high-level explanation of how IT used, what is the effect of information. With this high-level description, we can formulate the principle of information use measurement and management. 
Principle 1. Effects of information use are the changes inside or outside the mind. We shall measure information use results by this change's characteristics, by their relations, and by changes compliance to humans demands.

We will restrict to effects outside minds in further material because objects under study are technical, organizational, and socio-organizational systems.

Principle 2. We can measure information use quality in the given system by characteristics of changes, caused by information Im, by this changes relation and their compliance to humans demands.

For such effects, measure $\delta(\mathrm{Im}, \mathrm{S})$ of information use (application) quality may have the form: $\delta(\operatorname{Im}, \mathrm{S})=\Psi(\operatorname{Im}, \mathrm{S})-\Psi(\mathrm{S})$, where $\Psi(\operatorname{Im}, \mathrm{S})$ - measure of the system capability as system potential measure [21] under assumption information Im obtained with the use of IT It used to change (alter) system $S$ functioning and $\Psi(\mathrm{S})$ is system potential measure under assumption Im was not available (not obtained, not created, not constructed) because of other IT used. Obtaining information actions are classified in figure 2.

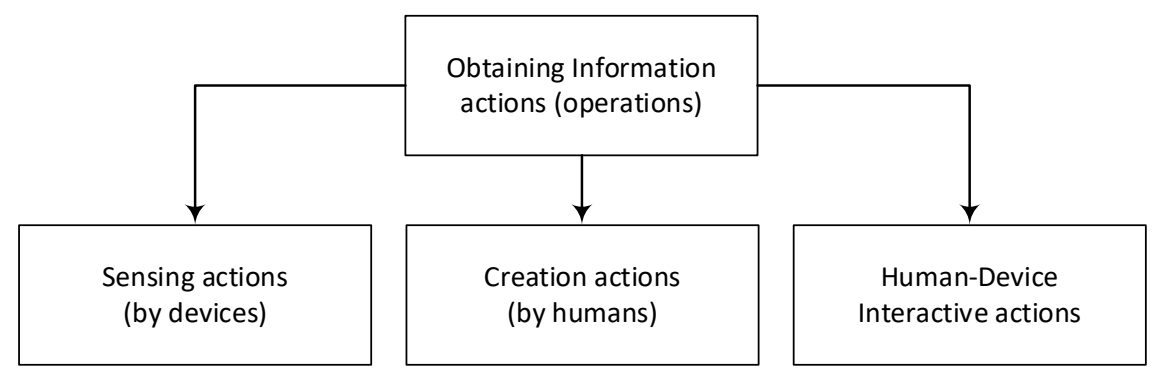

Fig. 2. Information retrieval actions classification. Second level classification.

Obtaining information actions (operations) $a^{o}=\left\{a^{s}, a^{c}, a^{\text {hd }}\right\}$, where $a^{s}-$ sensing actions (output information provided by devices based on the material world outside the mind), $a^{c}$ - creation actions (output information created by humans), $a^{\text {hd }}-$ human-device interaction actions.

Let $\operatorname{Im}_{j}=f_{j}\left(a^{j}\right)$, where $a^{j}-$ system of information actions and $I m_{j}$ is output information of $a^{j}$, provided by $f_{j}\left(a^{j}\right)$. Then, $\Delta\left(a^{j}, \mathrm{~S}\right)=\Psi\left(f_{j}\left(a^{j}\right), \mathrm{S}\right)-\Psi(\mathrm{S})-$ measure of information operations $a^{j}$ quality. $a^{j}$ quality measured considering all possible actions $a^{j *}$ which results from $a^{j}$ till material world effects manifested. Measure construction discussed below.

After material world effects manifested, the new chain of information actions possible started obtaining information actions. As a result, sequences $\left\langle a_{1}{ }^{j *}, \ldots, a_{i}^{j *}, \ldots a_{n}^{j *}\right\rangle$ possible, where $a_{i}{ }^{j *}$ - subsequences of possible information operations which lead to possible changes in the material world. In general, these sequences may include sequences of information actions that cause changes in human minds. But finally, material world action change will happen.

We will use such material world actions changes as markers of each contour of information actions fulfillment. That results in the third principle.

Principle 3. The contour of possible changes due to information actions ends when material world changes occur. The possible contour of possible changes shall be measured by changes in practice (in the material world). Possible sequences of such contours shall be considered. So possible sequences of contours realizations shall be measured.

Thus, after results are obtained due to successive contour, the next possible contours of purposeful changes with further information actions shall be taken into consideration and measured in their sequences. Information processing operations are classified in figure 3. 
Information processing actions $a^{p}=\left\{a^{t s}, a^{t}, a^{c}\right\}$, where $a^{t s}$ - time displacement and space moving actions, $a^{t}$ - form transformation actions, $a^{c}$ - creation of new information based on current information. Let $a^{j p}=a^{p}\left(a^{j}\right), a^{j}, f_{j p}=f_{p}\left(a^{p}\right) \circ f_{j}\left(a^{j}\right)$. Then, $\Delta\left(a^{j p}, \mathrm{~S}\right)-$ measure of information operations $a^{p}$ quality. $a^{j}$ quality measured considering all possible actions $a^{j p *}$ which results from $a^{j p}$ until material world effects manifested, then - next possible contours of purposeful changes with further information actions shall be considered. Information use operations are classified in figure 4.

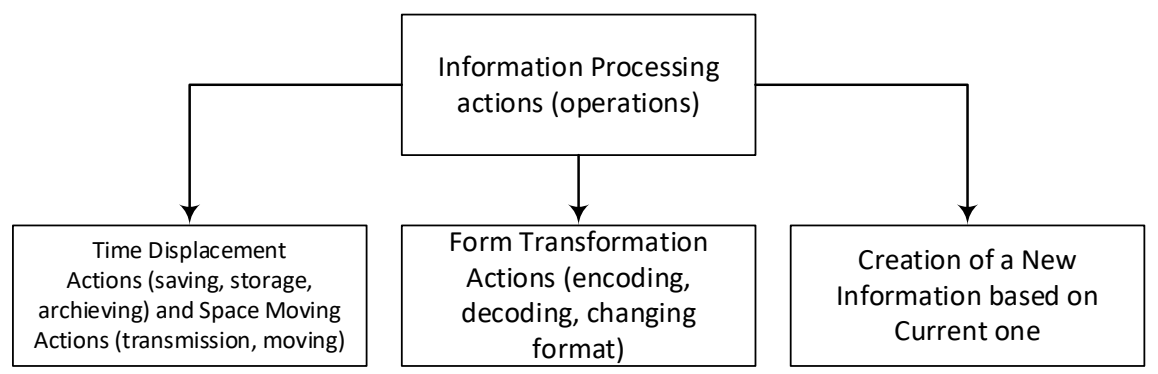

Fig. 3. Information processing operations. Second level classification.

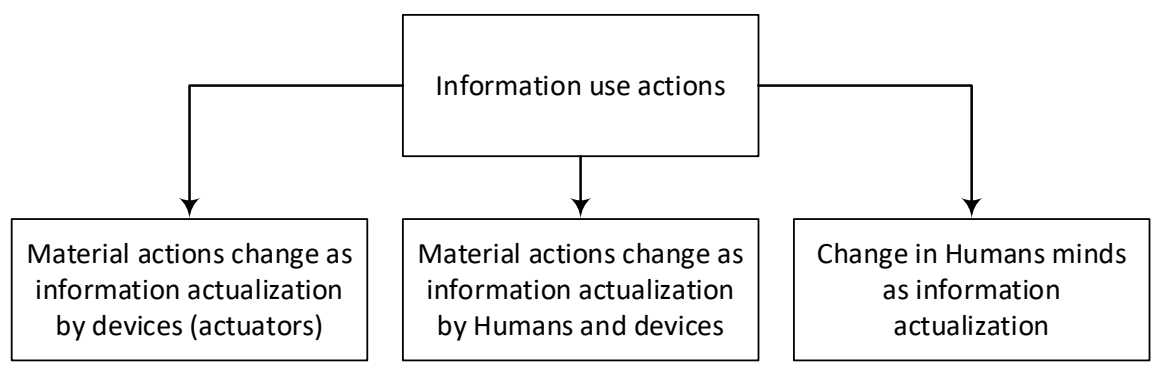

Fig. 4. Information use action classification. Second-level classification.

Information use actions $a^{u}=\left\{a^{a}, a^{h d}, a^{m}\right\}$, where $a^{d}$ - change in the actions of the material world as information actualization by devices (actuators), $a^{\text {hd }}$ - change in the actions of the material world as information actualization by human labor and equipment, $a^{m}-$ creation of new information based on current information. Let $a^{j p u}=a^{u}\left(a^{p}\right), a^{p}\left(a^{j}\right), a^{j}$, $f_{j p u}=f_{u}\left(a^{u}\right) \circ f_{p}\left(a^{p}\right) \circ f_{j}\left(a^{j}\right)$. Then, $\Delta\left(a^{j p u}, \mathrm{~S}\right)$ - measure of information operations $a^{u}$ quality. $a^{u}$ quality measured considering all possible actions $a^{j p u *}$ which results from $a^{u}$ taking into account the next contours of purposeful changes with the use of further information actions. Each contour of purposeful changes quality estimated by its final $\Delta\left(a^{j p u}, \mathrm{~S}\right)$ and probability $\mathrm{P}\left(a^{j p u}, \mathrm{~S}\right)$ of this contour realization. Each of the previous information actions quality estimated as results of corresponding $a^{j p u}, \mathrm{~S}$ possible after these information actions.

To estimate each chain $C_{n}$ of contours corresponding measure $\omega\left(C_{n}\right)$ constructed.

For example, we can measure each contour as:

$$
\omega\left(C_{n}, S\right)=<P\left(C_{n}\right), \Delta\left(a^{j p u}, S\right), n \in N>,
$$

Where $P\left(C_{n}\right)=P\left(a_{n}{ }^{j *}, S\right) \ldots P\left(a_{n}{ }^{j p u}, S\right), a_{n}{ }^{j p u_{-}}$final system of contour $C_{n}$ material world actions change, $P\left(a_{n}{ }^{j *}, S\right)$ - first information actions set in the contour of purposeful changes. For all possible $n \in N$ multidimensional measure $\Omega(S, I t)$ constructed, where ItIT used in the system $S$. Measure $\Omega(S, I t)$ characteristics (quantiles, moments, mixtures of characteristics) may serve as system potential vector $\Psi(S, I t)$ or scalar $\psi(S, I t)$ indicators.

For example, if the mean of $\Omega(S, I t)$ used: 


$$
\psi(S, I t)=\sum_{n=1}^{N} P\left(C_{n}\right), \Delta\left(a^{j p u}, \mathrm{~S}\right) .
$$

Measures $(1,2)$ can be used to estimate indicators of other properties, which characterize various aspects of the system change quality. Other measures used and examples of other properties estimation, such as dynamic capability, organizational capability, IT capability indicators estimation, can be found at [21-25].

\section{Data structures examples to estimate system potential}

The actions names and actions modes names and parameters table example shown in Table 1. All actions of networks are united into one table. The correspondence of network vertices ID's and actions ID's is given in Table 1.

Table 1. The actions and action modes data

$\begin{array}{llllll}a_{i} & a_{m} i & t_{a} & t_{b} & \ldots & \text { Name } \\ 1 & 1 & 3 & 5 & \ldots & \text { Conn. oftheP921AtolA } \\ 2 & 1 & 8 & 30 & \ldots & \text { Conn. oftheP27ABtolA } \\ \ldots & \ldots & \ldots & \ldots & \ldots & \ldots \\ n & 1 & 12 & 55 & \ldots & \text { FuelingoftheP921A } \\ \ldots & \ldots & \ldots & \ldots & \ldots & \ldots\end{array}$

The structures shown allow the creation of possible sequences of environment state changes. Each of such sequences causes the sequence of networks to be performed in response to the environment changes. Examples of such sequences in the tabular form shown at Table 2.

Table 2. Alternative Sequences of Environment States in Time.

\begin{tabular}{|l|l|l|l|l|l|l|l|}
\hline № & $\left\langle c_{i}^{e}\right\rangle / T_{z}$ & $T_{0}$ & $T_{1}$ & $T_{2}$ & $\ldots$ & $T_{j}$ & $\ldots$ \\
\hline & $\left\langle c_{0}^{e}\right\rangle$ & $c_{0}^{e}$ & $c_{0}^{e}$ & $c_{0}^{e}$ & & $c_{0}^{e}$ & \\
\hline & $\left\langle c_{0}^{e}, c_{1}^{e}\right\rangle$ & $c_{0}^{e}$ & $c_{0}^{e}$ & $c_{0}^{e}$ & & $c_{1}^{e}$ & \\
\hline$\ldots$ & $\ldots$ & $c_{0}^{e}$ & $c_{0}^{e}$ & $c_{1}^{e}$ & $\ldots$ & $c_{1}^{e}$ & $\ldots$ \\
\hline $\mathrm{n}$ & $\left\langle c_{0}^{e}, c_{1}^{e}, c_{2}^{e}\right\rangle$ & $c_{0}^{e}$ & $c_{1}^{e}$ & $c_{1}^{e}$ & & $c_{2}^{e}$ & \\
\hline$\ldots$ & $\ldots$ & $c_{0}^{e}$ & $c_{1}^{e}$ & $c_{2}^{e}$ & $\ldots$ & $c_{2}^{e}$ & $\ldots$ \\
\hline
\end{tabular}

The network alternations are described with the corresponding network cut ID's. The cut is the set of vertices that serve as the one network's borderline to its alternation. Corresponding alternative sequences of the environment changes and network cuts in the sequences are shown in Table 3.

Table 3. Alternative Sequences of the Environment Changes and Corresponding Network Cuts

$\begin{array}{llll}\text { SID } & \text { CID } & \text { ChangeID } & \text { ProbA } \\ 23527897 & 321 & 432 & 0,0000125 \\ 23527898 & 322 & 432 & 0,0000117 \\ 23527899 & 323 & 432 & 0,0000119\end{array}$

Each SID corresponds to different sequences of cuts referred by CID. ChangeID is environment change ID, and ProbA is the probability of sequence actualization. Between each pair of cuts, two networks inserted - i.e., remedial network from one goal to another and next, network to achieve a new goal. Each sequence of networks cuts and corresponding networks between cuts then translated into sequences of ordered operations in the lexicographic order. Such order determines acceptable structures for serial computations in multidimensional arrays created. The intermediate structure used to determine the sequence of functions computation is shown in Table 4. Information operations provide functions that 
map the environment and other conditions to possible alternations of networks. Example of the table that provides functional dependencies of the environment's state changes at the given moments and changes the demands to the system's functioning shown in Table 5.

Table 4. The correspondence of possible networks structures and actions ID's data

$\begin{array}{llllll}V_{i} & N_{3} & N_{4} & N_{5} & N_{6} & N_{7} \\ 1 & 1 & 1 & 26 & 1 & 1 \\ 2 & 2 & 2 & 27 & 2 & 2 \\ 3 & 3 & 25 & 25 & 25 & 3 \\ 4 & 4 & 4 & 4 & 4 & 4 \\ 5 & 5 & 5 & 28 & 5 & 5 \\ \ldots & \ldots & \ldots & \ldots & \ldots & \ldots \\ 33 & \text { NULL } & \text { NULL } & \text { NULL } & 37 & 37\end{array}$

Table 5. Correspondence of alternation moments, environmental states changes and demands ID's to

\begin{tabular}{llllll}
\multicolumn{5}{c}{ the system functioning. } \\
$T_{z}$ & $C_{p}, C_{q}$ & $G_{n}, G_{m}$ & Dem 1 & Dem 2 & Dem 3 \\
1 & $C 1, C 2$ & $G 1, G 2$ & 1 & 38 & 75 \\
2 & $C 1, C 2$ & $G 1, G 2$ & 2 & 39 & 76 \\
3 & $C 1, C 2$ & $G 1, G 2$ & 3 & 40 & 77 \\
$\ldots$ & $\ldots$ & $\ldots$ & $\ldots$ & $\ldots$ & $\ldots$ \\
37 & $C 1, C 2$ & $G 1, G 2$ & 37 & 74 & 111
\end{tabular}

\section{Examples of the system potential computation results}

Multidimensional measure $\Omega(S, I t)$ - probabilities computations results - shown below in figures 5-7. Figure 5 shows a $3 \mathrm{D}$ histogram, which offers some cases of $C_{n}$ realizations depending on actualization probabilities and measures of correspondence computed.

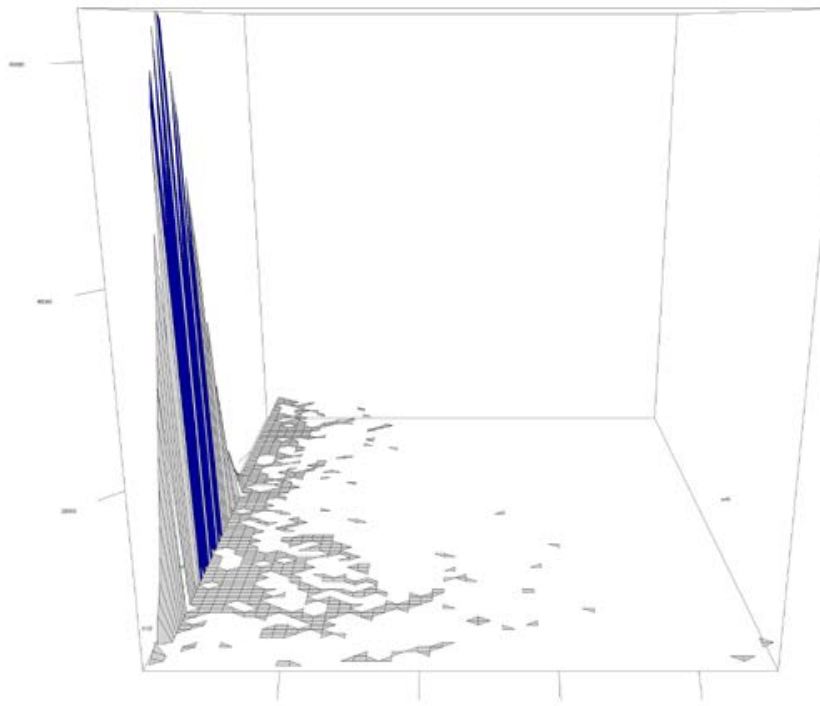

Fig. 5. 3D histogram which shows some cases of $C_{n}$ realizations depending slots of actualization probabilities and measures of correspondence.

Multidimensional measure $\Omega(S, I t)$ surface extrapolation (by kriging method) for contours of length two shown in figure 6 and for contours of length three - at figure 7. 


\section{Conclusion}

Using suggested concepts, models, and methods, researchers can apply system capability, dynamic capability, and organizational capability investigation regarding information technology use. Prescriptive and predictive modeling of information technologies use results becomes possible.

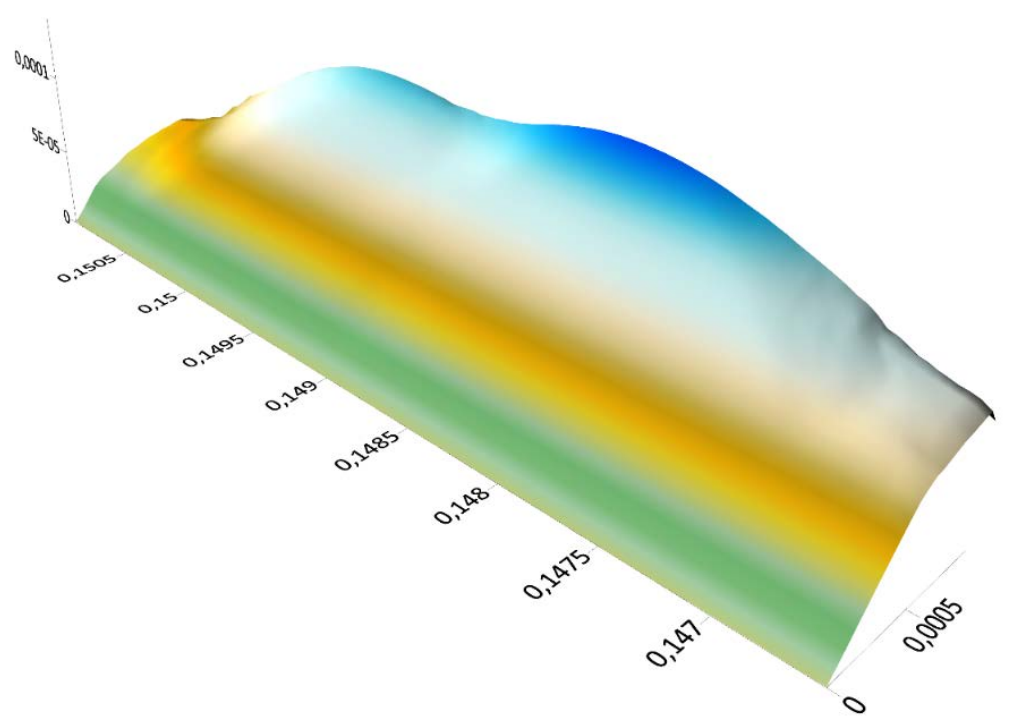

Fig. 6. 3D probability surface extrapolation (by kriging method) for contours of length two

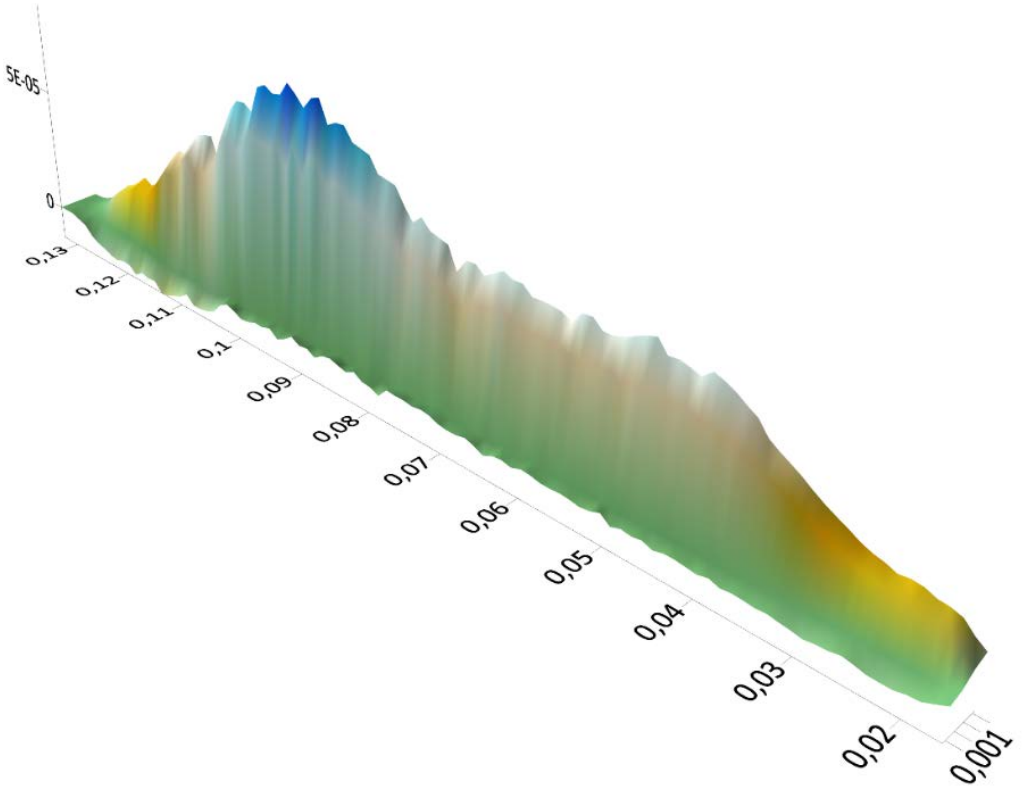

Fig.7. 3D probability surface extrapolation (by kriging method) for contours of length three 
The reported study was funded by RFBR, project number 20-08-00649 and 19-08-00989.

\section{References}

1. Felix Arndt, Ryan Bruce Gould. Revisiting Dynamic Capabilities through the lens of Complexity Theory // 24th Australian New Zealand Academy of Management Conference. Adelaide, Australia. (2010)

2. Di Stefano, G. Dynamic capabilities deconstructed: a bibliographic investigation into the origins, development, and future directions of the research domain / G. Di

Stefano, M. Peteraf, G. Verona // Long Range Planning. Vol.19, №4. - P.1187-1204. (2010)

3. Dixon, S. Building Dynamic Capabilities of Adaptation and Innovation / S. Dixon, K. Meyer, M. Day // Long Range Planning. Vol.47. - P.186-205. (2014)

4. The Digital Path to Business Resilience / Karalee Close, Michael Grebe, Phillip Andersen [et al.],URL:https:/www.bcg.com/publications/2020/digital-path-tobusiness-resilience. (2020)

5. Mikalef, P. Information technology-enabled dynamic capabilities and their indirect effect on competitive performance / P. Mikalef, A. Pateli // Journal of Business Research. Vol.70. - P. 1-16. (2017)

6. Mikalef, P. Developing IT-Enabled Dynamic Capabilities / P. Mikalef // Lecture Notes in Business Information Processing: Springer International Publishing, P.87100. (2014)

7. van de Wetering, R.A strategic alignment model for IT flexibility and dynamic capabilities $/ / 25^{\text {th }}$ European Conference on Information Systems (ECIS). Guimarães, Portugal, 2017.

8. Andrew McAfee.Machine, Platform, Crowd: Harnessing Our Digital Future / Andrew McAfee, Erik Brynjolfsson: W. W. Norton \& Company (2018)

9. Brynjolfsson, E. Beyond the Productivity Paradox / E. Brynjolfsson, L.M. Hitt // Communications of the ACM. Vol 44, № 8.c (1998)

10. Warner, K.S.R. Building dynamic capabilities for digital transformation / K.S.R. Warner, M. Wäger // Long Range Planning, Vol.52. - P.326-349 (2019).

11. Laney, D.B.Infonomics [electronic resource] / D.B. Laney. - Milton: Routledge, 2017.

12. Brand, K.IT Governance based on CobiT 4.1 / K. Brand, H. Boonen. - [Zaltbommel]: Van Haren, 150 p. (2010)

13. Duberry, J. Global environmental governance in the information age / J. Duberry. - London, New York: Routledge Taylor \& Francis Group (2019)

14. Parida, V. Reviewing Literature on Digitalization, Business Model Innovation, and Sustainable Industry: Past Achievements and Future Promises / V. Parida, D. Sjödin, W. Reim // Sustainability. Vol.11, № 2. - P. 391. (2019)

15. Portinale, L.Modeling and analysis of dependable systems: A probabilistic graphical mo del perspective / L. Portinale, D.C. Raiteri. - New Jersey: World Scientific, 2015.

16. Karna, A.Revisiting the role of the environment in the capabilities-financial performance relationship: A meta-analysis / A. Karna, A. Richter, E. Riesenkampff // Strategic Management Journal. Vol.37, №6. - P.1154-1173 (2016).

17. Torres, P.M.d.A.An overview on strategic design for socio-technical innovation / P.M.d.A. Torres // Strategic Design Research Journal. Vol.11, №3 (2018)

18. Sugarman, B. Dynamic Capability Seen through a Duality-Paradox Lens / B. Sugarman // Research in Organizational Change and Development: Emerald Group Publishing Limited. -P.141-189 (2014) 
19. Simon, H.A. Bounded Rationality and Organizational Learning / H.A. Simon // Organization Science. - Vol.2. - P.125-134 (1991)

20. Aslaksen, E.W. The System Concept and Its Application to Engineering / E.W. Aslaksen. - Berlin, Heidelberg: Springer Berlin Heidelberg, (2013)

21. Geyda, A.S. Information technology capability analytical research example/ A.S. Geyda: IDIMT 2020: Digitalized Economy, Society and Information Management 28th Interdisciplinary Information Management Talks, p.p 67-74 (2020)

22. Geyda, A. System Potential Estimation with Regard to Digitalization: Main Ideas and Estimation Example / A. Geyda, I. Lysenko // Information.Vol.11, №3. - P.164. (2020)

23. Geyda, A. Analytical Research on System Capability and Information Technology Use Capability: Problem Statement Examples / A. Geyda // 2020 26th Conference of Open Innovations Association (FRUCT): 2020 26th Conference of Open Innovations Association (FRUCT): IEEE, 20.04.2020 - 24.04.2020. - p.p.1-9. (2020)

25. Geyda, A.S. Complex of Models for System Capability Estimation with Regard to Information Technology Use / A.S. Geyda // AMCIS 2020 Proceedings. - p.6. (2020) 